\title{
High-Performance Battery-Pack Power Estimation Using a Dynamic Cell Model
}

\author{
Gregory L. Plett, Senior Member, IEEE
}

\begin{abstract}
In some battery applications, such as in hybrid electric vehicles or battery electric vehicles, it is necessary to be able to estimate, in real time, the present available power that may be sourced by the battery pack. Similarly, in rechargeable packs, it may be necessary to know how much charging power the pack can accept. These values must be carefully calculated in such a way that the pack will not be damaged by over/under charge or voltage or by exceeding a design current or power limit. This paper describes a method that uses a dynamic cell model and state-of-charge side information to very accurately predict the battery-pack available power.
\end{abstract}

Index Terms-Battery chargers, electric vehicle control, hybrid electric vehicle control, power control, road vehicle power systems.

\section{INTRODUCTION}

A NUMBER of high-performance battery applications require precise real-time estimates of the power available to be sourced by the battery pack: hybrid electric vehicles (HEVs) and battery electric vehicles (BEVs) are two examples. In both cases, the vehicle controller requires continuous up-to-date information from the battery-management system (BMS) regarding the power that may be supplied to the electric motor from the battery pack and power that may be supplied to the pack via regenerative braking or by active recharging via the motor.

Specifically, the problem we address in this paper may be described in the following way.

1) Discharge power: Based on present battery-pack conditions, estimate the maximum discharge power that may be maintained constant for $\Delta t \mathrm{~s}$ without violating preset operational design limits on cell voltage, state-of-charge (SOC), power, or current.

2) Charge power: Based on present battery-pack conditions, estimate the maximum battery-charge power that may be maintained constant for $\Delta t \mathrm{~s}$ without violating preset operational design limits on cell voltage, SOC, power, or current.

3) Both discharge and charge power: Any combination of 1) and 2), where $\Delta t$ may have different values for charge and discharge.

A standard method - in present use by battery-management systems for vehicular applications-is one that we will refer to as

Manuscript received December 1, 2003; revised May 6, 2004. This work was supported in part by Compact Power Inc. (CPI), Monument, CO.

The author is with the Department of Electrical and Computer Engineering, University of Colorado at Colorado Springs, Colorado Springs, CO 80918 USA and Compact Power Inc., Monument, CO 80132 USA (e-mail: glp@eas.uccs.edu, gplett@ compactpower.com).

Digital Object Identifier 10.1109/TVT.2004.832408 the hybrid pulse power characterization (HPPC) method specified by the Partnership for New Generation Vehicles (PNGV) [1] (described in Section II). In this paper, we compare the standard PNGV HPPC method with two new methods to be developed here.

The notation and assumptions we employ are as follows. We denote the number of cells in the battery pack by $n$; cell voltage for cell number $k$ in the pack by $v_{k}(t)$, which has operational design limits $v_{\min } \leq v_{k}(t) \leq v_{\max }$ that must be enforced for all $k: 1 \leq k \leq n$; SOC for cell number $k$ in the pack by $z_{k}(t)$, which has operational design limits $z_{\min } \leq z_{k}(t) \leq z_{\max }$ that must be enforced for all $k: 1 \leq k \leq n$; cell power by $p_{k}(t)$, which has operational design limits $p_{\min } \leq p_{k}(t) \leq p_{\max }$ that must be enforced for all $k: 1 \leq k \leq n$; and cell current by $i_{k}(t)$, which has operational design limits $i_{\min } \leq i_{k}(t) \leq i_{\max }$ that must be enforced for all $k: 1 \leq k \leq n$. Any particular limit may be removed if desired by replacing its value with $\pm \infty$, as appropriate. All limits $\left(v_{\max }, v_{\min }, z_{\max }, z_{\min }, i_{\max }, i_{\min }\right.$, $p_{\max }$, and $p_{\min }$ ) may furthermore be functions of temperature and other factors pertaining to the present battery-pack operating condition. Different cells may have different limits should this be necessary for any reason. Here, we assume that discharge current and power have positive sign and charge current and that power have a negative sign. Other sign conventions may be used and the method to be described may be adapted to these conventions in a forthright manner.

For simplicity of description, the battery pack is assumed to comprise $n_{s}$ cell modules connected in series, where each cell module comprises $n_{p}$ individual cells connected in parallel and $n_{s} \geq 1, n_{p} \geq 1$. Other configurations are possible and are accommodated by straightforward modifications to the way in which the method is described.

\section{PNGV HPPC METHOD}

In this section, we will describe the PNGV HPPC method, which considers operational design limits on voltage when estimating available power, but does not consider design limits on cell current, cell power, or cell SOC and only approximately applies a prediction horizon $\Delta t$ to the estimate. The HPPC method may be described by assuming that each cell in the battery pack is modeled by the approximate relationship

$$
v_{k}(t)=\operatorname{OCV}\left(z_{k}(t)\right)-R \times i_{k}(t)
$$

where $\operatorname{OCV}\left(z_{k}(t)\right)$ is the open-circuit-voltage of cell $k$ at its present SOC and $R$ is a constant representing the cell's internal resistance. Different values of $R$ may be used for charge and 
discharge currents, if desired, and are denoted as $R^{\text {chg }}$ and $R^{\text {dis }}$, respectively. ${ }^{1}$

Since the design limits $v_{\min } \leq v_{k}(t) \leq v_{\max }$ must be enforced, we may calculate the maximum discharge current as constrained by voltage as

$$
i_{\max , k}^{\mathrm{dis}, \mathrm{volt}}=\frac{\mathrm{OCV}\left(z_{k}(t)\right)-v_{\min }}{R^{\mathrm{dis}}} .
$$

We may similarly calculate the maximum magnitude charge current based on voltage. Note, however, that charge current is assumed negative in sign by convention, so that the maximummagnitude current is a minimum in the signed sense, which is

$$
i_{\min , k}^{\text {chg,volt }}=\frac{\operatorname{OCV}\left(z_{k}(t)\right)-v_{\max }}{R^{\text {chg }}} .
$$

Pack power is then calculated as

$$
\begin{aligned}
& P_{\min }^{\mathrm{chg}}=n_{s} n_{p} \max _{k}\left(v_{\max } i_{\text {min }, k}^{\text {chg,volt }}\right) \\
& P_{\max }^{\text {dis }}=n_{s} n_{p} \min _{k}\left(v_{\min } i_{\text {max }, k}^{\text {dis, volt }}\right) .
\end{aligned}
$$

The HPPC method is limited in several respects. First, as we have noted, there are neither SOC, maximum current, nor maximum power design constraints involved in the computation. More importantly, the cell model used is too primitive to give precise results. Overly optimistic or pessimistic values could be generated, either posing a safety or battery-health hazard or being inefficient in battery use. A better cell model, combined with a maximum-power algorithm that uses the cell model, can give better power prediction. Here, we explore this idea and propose two improved methods. Method I will be developed in Sections III and IV and method II will be developed in Sections III and $\mathrm{V}$.

\section{RATE LIMITS BASED ON SOC}

The first aspect of the new methods is the addition of SOC limits to the power calculation and the explicit inclusion of a time horizon $\Delta t$. (Limits based on cell power and current design limits will be added later in a very straightforward way.) This may be done as follows. First, for a constant current $i_{k}$, the SOC recurrent relationship is

$$
z_{k}(t+\Delta t)=z_{k}(t)-\left(\frac{\eta_{i} \Delta t}{C}\right) i_{k}
$$

where $z_{k}(t)$ is the present SOC for cell $k, z_{k}(t+\Delta t)$ is the predicted SOC $\Delta t \mathrm{~s}$ into the future, $C$ is the cell capacity in ampere-seconds, and $\eta_{i}$ is the Coulombic efficiency factor at current level $i_{k}$. Here, we assume that $\eta_{i}=1$ for discharge currents and $\eta_{i}=\eta \leq 1$ for charge currents.

If we have design limits on SOC such that $z_{\min } \leq z_{k}(t) \leq$ $z_{\max }$ for all cells in the pack, then we can compute current $i_{k}$

\footnotetext{
${ }^{1}$ The HPPC procedure implements a crude prediction horizon, as we will see, by using values of $R^{\text {chg }}$ and $R^{\text {dis }}$ that have been modified somewhat from the true Ohmic resistance of the cell in order to approximate the 18-s discharge and the 2-s charge resistances. This approximation is not accurate over all states of charge, however.
}

such that these limits are not exceeded. Simple algebra gives limits based on the SOC of each cell

$$
\begin{aligned}
& i_{\max , k}^{\mathrm{dis}, \mathrm{soc}}=\frac{z_{k}(t)-z_{\min }}{\frac{\Delta t}{C}} \\
& i_{\min , k}^{\mathrm{chg}, \mathrm{soc}}=\frac{z_{k}(t)-z_{\max }}{\frac{\eta \Delta t}{C}} .
\end{aligned}
$$

The pack maximum currents—based only on cell SOC—-then are

$$
\begin{gathered}
i_{\max }^{\mathrm{dis}, \mathrm{soc}}=\min _{k}\left(i_{\mathrm{max}, k}^{\mathrm{dis}, \mathrm{soc}}\right) \\
i_{\min }^{\mathrm{chg}, \mathrm{soc}}=\max _{k}\left(i_{\mathrm{min}, k}^{\mathrm{chg}, \mathrm{soc}}\right) .
\end{gathered}
$$

This method assumes that we have a valid SOC estimate available for every cell in the pack. If this is not the case, then an approximate remedy would be to calculate

$$
\begin{aligned}
& i_{\max }^{\mathrm{dis,soc}} \approx \frac{z(t)-z_{\min }}{\frac{\Delta t}{C}} ; \\
& i_{\min }^{\mathrm{chg}, \mathrm{soc}} \approx \frac{z(t)-z_{\max }}{\frac{\eta \Delta t}{C}}
\end{aligned}
$$

where $z(t)$ is the pack SOC.

On the other hand, we might have more information available than simply cell SOC. For example, if we use a Kalman filtering method to estimate all cell SOCs in a pack, then we also have estimates of the uncertainty of the SOC estimate itself [2]-[7]. Let the uncertainty have Gaussian distribution with standard deviation, as estimated by the Kalman filter, denoted as $\sigma_{z}$. Then, we have $95.5 \%$ confidence that the true SOC is within the estimate $\pm 2 \sigma_{z}$ and we have $99.7 \%$ confidence that the true SOC is within the estimate $\pm 3 \sigma_{z}$. We can incorporate this information into the estimate of maximum current based on SOC to have very high confidence that SOC design limits will not be violated. This is done (assuming here that we desire to use a $3 \sigma_{z}$ confidence interval) as

$$
\begin{aligned}
i_{\max , k}^{\mathrm{dis}, \mathrm{soc}} & =\frac{\left(z_{k}(t)-3 \sigma_{z}\right)-z_{\min }}{\frac{\Delta t}{C}} \\
i_{\min , k}^{\mathrm{chg}, \mathrm{soc}} & =\frac{\left(z_{k}(t)+3 \sigma_{z}\right)-z_{\max }}{\frac{\eta \Delta t}{C}} .
\end{aligned}
$$

\section{Revised RATE Limits BASED ON Voltage-Method I: USING A SiMPle CELl MODEL}

The second aspect of the new methods corrects a limitation in the HPPC method for applying voltage limits. If we assume the cell model of (1) and that $R^{\text {chg }}$ and $R^{\text {dis }}$ are the cell's Ohmic resistances, then (2) and (3) predict the instantaneously available current, not the constant value of current that is available for the next $\Delta t \mathrm{~s}$. The result of this calculation, if applied to a scenario in which $\Delta t$ is large, poses a safety or battery-health issue, as the cells may become over/under charged. For the time being, retaining (1) for the cell model, the corrected prediction is achieved by

$$
v_{k}(t+\Delta t)=\operatorname{OCV}\left(z_{k}(t+\Delta t)\right)-R \times i_{k}(t)
$$


which cannot be directly solved in closed form for the maximum current $i_{k}$, since $z_{k}(t+\Delta t)$ is itself a function of current [cf. (4)] and $\operatorname{OCV}(\cdot)$ is a nonlinear relationship.

In this paper, we propose two methods to solve (7) for the maximum value of $i_{k}(t)$. The first uses a Taylor-series expansion to linearize the equation, so that we may solve for an approximate value of $i$. We assume that $\operatorname{OCV}(\cdot)$ is differentiable at point $z_{k}(t)$, which gives the result $[8$, Th.1]

$$
\begin{aligned}
\operatorname{OCV}\left(z_{k}(t+\Delta t)\right)= & \operatorname{OCV}\left(z_{k}(t)-i_{k} \frac{\eta_{i} \Delta t}{C}\right) \\
= & \operatorname{OCV}\left(z_{k}(t)\right)-i_{k}\left(\frac{\eta_{i} \Delta t}{C}\right) \\
& \times\left.\frac{\partial \operatorname{OCV}(z)}{\partial z}\right|_{z=z_{k}(t)} \\
& +R_{1}\left(z_{k}(t), i_{k} \frac{\left(\eta_{i} \Delta t\right)}{C}\right)
\end{aligned}
$$

where the first-order residual $R_{1}\left(z_{k}(t)\right.$, $\left.i_{k}\left(\eta_{i} \Delta t\right) / C\right) /\left\|z_{k}(t)\right\| \rightarrow 0$ as $i_{k}\left(\eta_{i} \Delta t\right) / C \rightarrow 0$ in $\mathbb{R}$. Note that the change in SOC over $\Delta t \mathrm{~s}$ is generally small, so we may approximate

$v_{k}(t+\Delta t) \approx \operatorname{OCV}\left(z_{k}(t)\right)-\left.i_{k} \frac{\eta_{i} \Delta t}{C} \frac{\partial \operatorname{OCV}(z)}{\partial z}\right|_{\substack{z=z_{k}(t) \\-R \times i_{k}}}$

which gives

$$
\begin{aligned}
i_{\mathrm{max}, k}^{\mathrm{dis}, \text { volt }} & =\left(\frac{\mathrm{OCV}\left(z_{k}(t)\right)-v_{\min }}{\left.\frac{\Delta t}{C} \frac{\mathrm{OOCV}(z)}{\partial z}\right|_{z_{k}(t)}+R^{\mathrm{dis}}}\right) \\
i_{\mathrm{min}, k}^{\mathrm{chg}, \text { volt }} & =\left(\frac{\mathrm{OCV}\left(z_{k}(t)\right)-v_{\mathrm{max}}}{\left.\frac{\eta \Delta t}{C} \frac{\partial \mathrm{OCV}(z)}{\partial z}\right|_{z_{k}(t)}+R^{\mathrm{chg}}}\right) .
\end{aligned}
$$

Both the function $\operatorname{OCV}(z)$ and its derivative $\partial \operatorname{OCV}(z) / \partial z$ might be computed from some known mathematical relationship for $\mathrm{OCV}(z)$, (e.g., , Nernst's equation) using either analytic or numeric methods or by a table lookup of empirical data. This quantity is positive for most battery electrochemistries over the entire SOC range, so the values computed by (8) and (9) are smaller in magnitude than those from (2) and (3) for the same values of $R^{\text {dis }}$ and $R^{\text {chg }}$. The HPPC procedure compensates for its inaccuracy by using modified values of $R^{\text {dis }}$ and $R^{\text {chg }}$, determined experimentally, that approximate the denominator terms in (8) and (9). We see that this cannot be accurate over the entire SOC range, however, as $\partial \mathrm{OCV}(z) / \partial z$ is not constant, particularly near extreme values of $z$.

It is important to note that the denominator terms in (8) and (9) depend on individual cell capacities and resistances, which vary somewhat from cell to cell, even in high-volume production. Additionally, these variations are likely to increase as the cells age. The computations involved may be quite sensitive to changes in the resistance term, but are relatively insensitive to changes in the capacity term, as $\Delta t$ is often on the order of $1 / 3600$ (for sampling rates of around $1 \mathrm{~Hz}$ ). Fortunately, it is simple to maintain an adaptive estimate of each cell's resistance and capacity, which is the topic of other research [4], for use in (8) and (9), if it proves necessary to do so. Sensitivity to SOC estimation error is relatively low since $\operatorname{OCV}\left(z_{k}\right)$ tends to be quite flat except at extreme SOC values, where power is limited by SOC in any case and the values computed by (8) and (9) are not relevant.

Once all of the cell current limits have been calculated, the pack discharge and charge currents with all limits enforced are computed as

$$
\begin{aligned}
i_{\max }^{\mathrm{dis}} & =\min \left(i_{\max }, \min _{k} i_{\max , k}^{\mathrm{dis}, \mathrm{soc}}, \min _{k} i_{\max , k}^{\mathrm{dis}, \mathrm{volt}}\right) \\
i_{\min }^{\mathrm{chg}} & =\max \left(i_{\min }, \max _{k} i_{\min , k}^{\mathrm{chg}, \mathrm{soc}}, \max _{k} i_{\min , k}^{\mathrm{chg}, \mathrm{volt}}\right)
\end{aligned}
$$

and power may be calculated using the sum of all cell powers. These are equal to the product of the maximum allowed current and the predicted future voltage.

$$
\begin{aligned}
& P_{\min }^{\mathrm{chg}}=n_{p} \sum_{k=1}^{n_{s}} i_{\min }^{\mathrm{chg}} v_{k}(t+\Delta t) \\
& \approx n_{p} \sum_{k=1}^{n_{s}} i_{\min }^{\operatorname{chg}}\left(\operatorname{OCV}\left(z_{k}(t)-i_{\min }^{\operatorname{chg}} \frac{\eta_{i} \Delta t}{C}\right)\right. \\
& \left.-R^{\mathrm{chg}} \times i_{\min }^{\mathrm{chg}}\right) \\
& P_{\max }^{\mathrm{dis}}=n_{p} \sum_{k=1}^{n_{s}} i_{\max }^{\mathrm{dis}} v_{k}(t+\Delta t) . \\
& \approx n_{p} \sum_{k=1}^{n_{s}} i_{\max }^{\mathrm{dis}}\left(\operatorname{OCV}\left(z_{k}(t)-i_{\max }^{\mathrm{dis}} \frac{\Delta t}{C}\right)\right. \\
& \left.-R^{\mathrm{dis}} \times i_{\max }^{\mathrm{dis}}\right) .
\end{aligned}
$$

Maximum and minimum cell power limits may also be imposed in this calculation. Note that in all equations, $\operatorname{OCV}(z), C, v_{\max }$, $v_{\min }, z_{\max }, z_{\min }, i_{\max }, i_{\min }, R^{\mathrm{chg}}$, and $R^{\mathrm{dis}}$ may be functions of temperature and other factors pertaining to the present battery pack operating conditions.

\section{Revised Rate Limits Based on Voltage-Method II: USING A COMPREHENSIVE CELL MODEL}

The method for solving (7) presented in Section IV is appropriate when the battery-management-system computational capability is low. A second method may be used when this is not a significant constraint. This second method assumes a more precise mathematical model of cell dynamics, which might be in a discrete-time state-space form, such as the following coupled pair of equations [9]:

$$
\begin{aligned}
x_{k}[m+1] & =f\left(x_{k}[m], u_{k}[m]\right) \\
v_{k}[m] & =g\left(x_{k}[m], u_{k}[m]\right)
\end{aligned}
$$

where $m$ is the discrete time sample index, the vector function of time $x_{k}[m]$ is called the "state" of the system, $u_{k}[m]$ is the input to the system, which includes cell current $i_{k}[\mathrm{~m}]$ as a component and might also include temperature, resistance, capacity and so 
forth, and $f(\cdot)$ and $g(\cdot)$ are functions chosen to model the cell dynamics. Alternate model forms, including continuous-time state-space forms and differential or difference equations might also be used. We assume that there is a method to compute SOC given the model that is implemented.

For convenience of presentation, here we assume that the cell model is in a discrete-time state-space form. Also assume that $\Delta t \mathrm{~s}$ may be represented in discrete time as $T$ sample intervals. Then, we can use this model to predict cell voltage $\Delta t \mathrm{~s}$ into the future by

$$
v_{k}[m+T]=g\left(x_{k}[m+T], u_{k}[m+T]\right)
$$

where $x_{k}[m+T]$ may be found by simulating (14) for $T$ time samples. We assume that the input remains constant from time index $m$ to $m+T$, so if temperature change, for example, over this interval is significant, it must be included as part of the dynamics modeled by (14) and not as a part of the measured input $u_{k}[m]$

The method then uses a bisection search algorithm [10], to be elaborated on in Section A, to find $i_{\max , k}^{\text {dis, volt }}$ and $i_{\min , k}^{\text {chg,volt }}$ by looking for the $i_{k}$ (as a member of the $u_{k}$ vector) that causes equality in

$$
\begin{aligned}
v_{\min } & =g\left(x_{k}[m+T], u_{k}[m+T]\right), \text { or } \\
0 & =g\left(x_{k}[m+T], u_{k}[m+T]\right)-v_{\min }
\end{aligned}
$$

to find $i_{\max , k}^{\mathrm{dis}, \mathrm{volt}}$, and by looking for the $i_{k}$ that causes equality in

$$
\begin{aligned}
v_{\max } & =g\left(x_{k}[m+T], u_{k}[m+T]\right), \text { or } \\
0 & =g\left(x_{k}[m+T], u_{k}[m+T]\right)-v_{\max }
\end{aligned}
$$

to find $i_{\mathrm{min}, k}^{\mathrm{chg}, \mathrm{volt}}$. A special case is when the state (14) is linear; that is, when

$$
x_{k}[m+1]=A x_{k}[m]+B u_{k}[m]
$$

where $A$ and $B$ are constant matrices. The model presented in Section VI is an example in which this is the case. Then, for input $u_{k}$ constant over the entire prediction horizon (from time $m$ to $m+T$ ), we have

$$
x_{k}[m+T]=A^{T} x_{k}[m]+\left(\sum_{j=0}^{T-1} A^{T-1-j} B\right) u_{k} .
$$

Most of these terms may be precomputed without knowledge of $u_{k}$ in order to speed calculation using the bisection algorithm.

Once the SOC-based current limits $i_{\max , k}^{\mathrm{dis}, \mathrm{soc}}$ and $i_{\mathrm{min}, k}^{\mathrm{chg}, \mathrm{soc}}$ are computed using (5) and (6) and the voltage-based current limits $i_{\max , k}^{\text {dis, volt }}$ and $i_{\text {min, }, k}^{\text {chet }}$ are computed using (16) and (17), overall current limits may be computed using (10) and (11). Power is then computed as

$$
\begin{aligned}
P_{\min }^{\mathrm{chg}} & =n_{p} \sum_{k=1}^{n_{s}} i_{\min }^{\operatorname{chg}} v_{k}(t+\Delta t) \\
& =n_{p} \sum_{k=1}^{n_{s}} i_{\min }^{\operatorname{chg}} g_{k}\left(x_{k}(m+T), u_{k}\right)
\end{aligned}
$$

with $u_{k}$ containing $i_{\text {min }}^{\text {chg }}$ as its value for current and

$$
\begin{aligned}
P_{\max }^{\mathrm{dis}} & =n_{p} \sum_{k=1}^{n_{s}} i_{\max }^{\mathrm{dis}} v_{k}(t+\Delta t) \\
& =n_{p} \sum_{k=1}^{n_{s}} i_{\max }^{\mathrm{dis}} g_{k}\left(x_{k}(m+T), u_{k}\right)
\end{aligned}
$$

with $u_{k}$ containing $i_{\max }^{\mathrm{dis}}$ as its value for current.

\section{A. Bisection Search}

To solve (16) and (17), we require a method to solve for a root of a nonlinear equation. Here, we use the bisection search algorithm to do so. The bisection search algorithm looks for a root of $f(x)$ (i.e., a value of $x$ such that $f(x)=0$ ) where it is known a priori that the root lies between values $x_{1}<$ root $<$ $x_{2}$. One way of knowing that a root lies in this interval is that the sign of $f\left(x_{1}\right)$ is different from the sign of $f\left(x_{2}\right)$.

Each iteration of the bisection algorithm evaluates the function at the midpoint $x_{\text {mid }}=\left(x_{1}+x_{2}\right) / 2$. Based on the sign of the evaluation, either $x_{1}$ or $x_{2}$ is replaced by $x_{\text {mid }}$ to retain different signs on $f\left(x_{1}\right)$ and $f\left(x_{2}\right)$. We see that the uncertainty in the location of the root is halved by this algorithmic step. The bisection algorithm repeats this iteration until the interval between $x_{1}$ and $x_{2}$ and, hence, the resolution of the root of $f(x)$ is as small as desired. If $\varepsilon$ is the desired root resolution, then the algorithm will require at most $\left\lceil\log _{2}\left(\left|x_{2}-x_{1}\right| / \varepsilon\right)\right\rceil$ iterations. The bisection method is listed in Algorithm 1.

Algorithm 1 The bisection algorithm [10] begin $\{$ bisection algorithm

set $x_{1}=$ first search limit

set $x_{2}=$ second search limit

set $\varepsilon=$ desired resolution on current in bisection output set JMAX = maximum number of bisection iterations

$$
\geq\left\lceil\log _{2}\left(\frac{\left|x_{2}-x_{1}\right|}{\varepsilon}\right)\right\rceil
$$

let func $(\cdot)$ be the function in which to find root set the search interval $d x=x_{2}-x_{1}$

if (func $\left.\left(\mathrm{x}_{1}\right) \geq 0\right)\left\{/ /\right.$ constraint : func $\left(\mathrm{x}_{1}\right)<0$

$$
\begin{aligned}
& d x=-d x \\
& x_{1}=x_{2}
\end{aligned}
$$

\}$/ /$ root is now between $\left(x_{1}, x_{1}+d x\right)$ and func $\left(x_{1}\right)<0$

for $j=1$ to JMAX \{

$d x=0.5 \times d x$

$x_{\text {mid }}=x_{1}+d x$

if $\left(\right.$ func $\left.\left(x_{\text {mid }}\right) \leq 0\right)\left\{x_{1}=x_{\text {mid }}\right\}$

if $(|d x| \leq \varepsilon) \quad\left\{\right.$ return $\left.\left(x_{1}+0.5 \times d x\right)\right\}$

\}//we have bisected too many times, so return best guess return $\left(x_{1}+0.5 \times d x\right)$

end $\{$ bisection algorithm $\}$

\section{B. Finding the Maximum/Minimum Current}

To determine maximum discharge and charge current for any particular cell, bisection is performed on (16) and (17). Bisection is incorporated in the overall algorithm as follows. First, three simulations are performed to determine cell voltages $\Delta t \mathrm{~s}$ 
into the future for cell current $i_{k}=0, i_{k}=i_{\min }$, and $i_{k}=i_{\max }$. If cell voltages are predicted to be between $v_{\min }$ and $v_{\max }$ for the maximum dis/charge rates, then these maximum rates may be used. If the cell voltages, even during rest, are outside of bounds, then set the maximum rates to zero. Otherwise, we know that the true maximum rate may be found by bisecting between rate equal to zero and its maximum value. Bisection is performed between current limits $\left(i_{\min }, 0\right)$ or $\left(0, i_{\max }\right)$.

\section{EXAMPle OF THE CELl Model}

In order to examine and compare performance of the PNGV HPPC method versus the two methods presented in this paper, we must first define a discrete-time state-space model of the form of (14) and (15) that applies to battery cells. Here, we briefly review the "enhanced self-correcting cell model" from [3] and [7]. This model includes effects due to open-circuit voltage, internal resistance, voltage time constants, and hysteresis. As an example, we fit parameter values to this model structure to model the dynamics of high-power lithium-ion polymer battery (LiPB) cells, although the structure and methods presented here are general.

The state of charge is captured by one state of the model. This equation is

$$
z_{k}[m+1]=z_{k}[m]-\left(\frac{\eta_{i} \Delta T}{C}\right) i_{k}[m]
$$

where $\Delta T$ represents the intersample period (in seconds) and $C$ represents the cell capacity (in ampere-seconds).

The time constants of the cell voltage response are captured by several filter states. If we let there be $n_{f}$ time constants, then

$$
f_{k}[m+1]=A_{f} f_{k}[m]+B_{f} i_{k}[m] .
$$

The matrix $A_{f} \in \mathbb{R}^{n_{f} \times n_{f}}$ may be a diagonal matrix with realvalued entries. If so, the system is stable if all entries have magnitude less than one. The vector $B_{f} \in \mathbb{R}^{n_{f} \times 1}$ may simply be set to $n_{f}$ " 1 "s. The value of $n_{f}$ and the entries in the $A_{f}$ matrix are chosen as part of the system-identification procedure to best fit the model parameters to measured cell data.

The hysteresis level is captured by a single state

$$
\begin{aligned}
h_{k}[m+1]= & \exp \left(-\left|\frac{\eta_{i} i_{k}[m] \gamma \Delta T}{C}\right|\right) h_{k}[m] \\
& +\left(1-\exp \left(-\left|\frac{\eta_{i} i_{k}[m] \gamma \Delta T}{C}\right|\right)\right) \operatorname{sgn}\left(i_{k}[m]\right)
\end{aligned}
$$

where $\gamma$ is the hysteresis rate constant, again found by system identification.

The overall model state is

$$
x_{k}[m]=\left[f_{k}[m]^{\prime} \quad h_{k}[m] \quad z_{k}[m]\right]^{\prime}
$$

where the prime symbol $\left({ }^{\prime}\right)$ is the matrix/vector transpose operator. The state equation for the model is formed by combining all of the individual equations above. Note that, at each time step, the state equation is linear in the input

$$
u_{k}[m]=\left[i_{k}[m] \quad 1\right]^{\prime}
$$

which speeds the prediction operation.

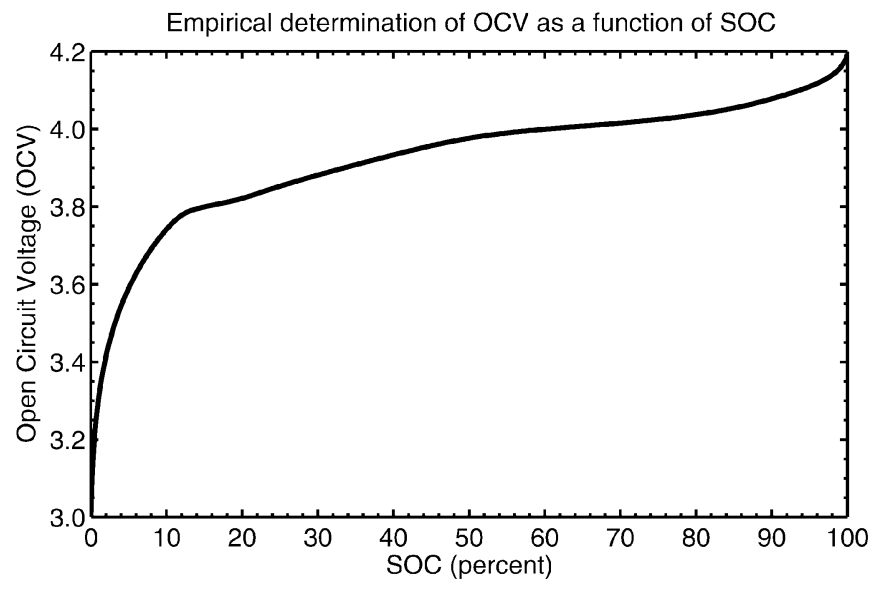

Fig. 1. Plot of open-circuit voltage as a function of the state of charge.

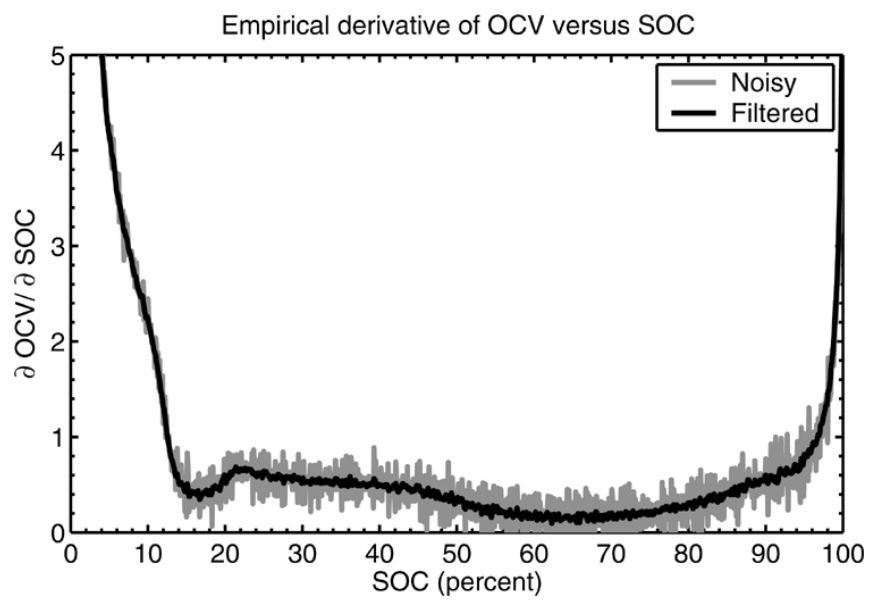

Fig. 2. Derivative of OCV as a function of SOC. The raw noisy version is shown as gray and the filtered derivative is shown as black.

The output equation that combines the state values to predict cell voltage is

$$
v_{k}[m]=\mathrm{OCV}\left(z_{k}[m]\right)+G f_{k}[m]-R i_{k}[m]+M h_{k}[m]
$$

where $G \in \mathbb{R}^{1 \times n_{f}}$ is a vector of constants that blend the timeconstant states together in the output, $R$ is the cell resistance (different values may be used for dis/charge), and $M$ is the maximum hysteresis level.

The open-circuit voltage as a function of the state of charge for these cells is plotted in Fig. 1. This is an empirical relationship found by cell testing. First, the cell was fully charged (constant current to $4.2 \mathrm{~V}$, constant voltage to $200 \mathrm{~mA}$ ). Then, the cell was discharged at the $\mathrm{C} / 25$ rate until fully discharged (3.0 $\mathrm{V})$. The cell was then charged at the $\mathrm{C} / 25$ rate until the voltage was $4.2 \mathrm{~V}$. The low rates were used to minimize the dynamics excited in the cells. The cell voltage as a function of state of charge under discharge and under charge were averaged to compute the OCV. This has the effect of eliminating, to the greatest extent possible, the presence of hysteresis and ohmic resistance in the final function. For the purpose of computations involving $\mathrm{OCV}$, the final curve was digitized at 200 points and stored in a table. Linear interpolation is used to look up values in the table.

The partial derivative of OCV with respect to SOC is plotted in Fig. 2. This relationship was computed by first taking finite 


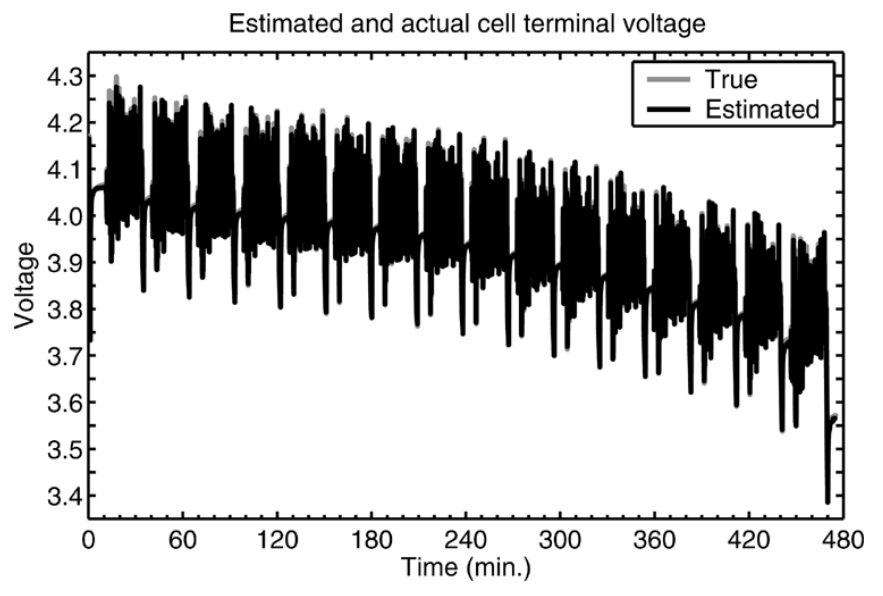

Fig. 3. Voltage prediction using the cell model. Gray is the true voltage and black is the estimated voltage.

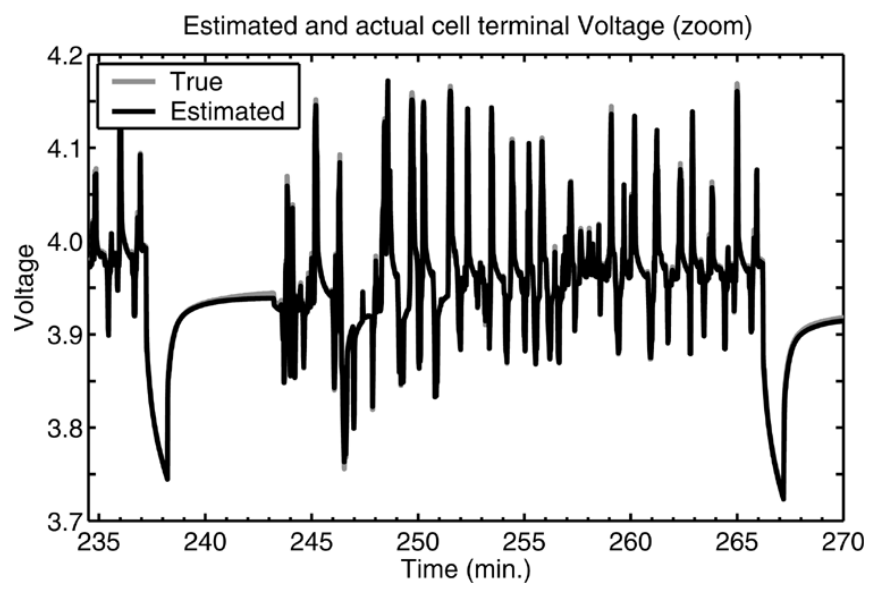

Fig. 4. Zoom of voltage prediction for one UDDS cycle at around 50\% SOC. Gray is the true voltage and black is the estimated voltage.

differences between points in the OCV plot in Fig. 1 and dividing by the distance between points (i.e., Euler's approximation to a derivative). The resulting data is too noisy to be of practical use, as shown in the gray line in Fig. 2. It was filtered using a zero-phase low-pass filter, resulting in the black line in Fig. 2, which may be used in the power calculation. This relationship is also digitized at 200 points and linear interpolation into the table of values is used when computations requiring this function are performed.

Other parameters are fit to the cell model. In particular, the model employs four low-pass filter states $\left(n_{f}=4\right)$, a nominal capacity of 7.5 Ah, and an intersample interval of $\Delta T=1 \mathrm{~s}$. There is very close agreement between the cell model voltage prediction and the cell true voltage. This is illustrated in Fig. 3. For this figure, the cell test was a sequence of 16 UDDS cycles, performed at room temperature, separated by discharge pulses and 5-min rests, and spread over the 90\%-10\% SOC range. The difference between true cell terminal voltage and estimated cell terminal voltage is very small [a root-mean-squared (rms) voltage estimation error of less than $5 \mathrm{mV}$ ]. To better illustrate the model's fidelity, refer to the zoom on one UDDS cycle in the 50\% SOC region, shown in Fig. 4. The SOC as a function of time is plotted in Fig. 5. We see that SOC increases by about 5\%

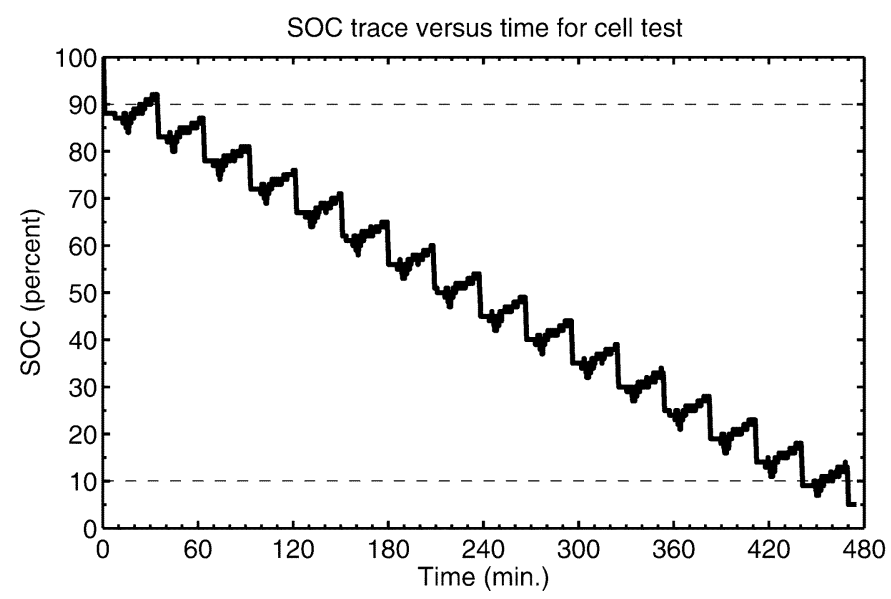

Fig. 5. SOC trace for cell test.

TABLE I

PARAMETERS FOR POWER-CALCULATION EXAMPLES

\begin{tabular}{ccc}
\hline Parameter & Minimum & Maximum \\
\hline$v_{k}(t)$ & $3.0 \mathrm{~V}$ & $4.35 \mathrm{~V}$ \\
$i_{k}(t)$ & $-200 \mathrm{~A}$ & $200 \mathrm{~A}$ \\
$z_{k}(t)$ & 0.1 & 0.9 \\
$p_{k}(t)$ & $-\infty$ & $\infty$ \\
\hline
\end{tabular}

during each UDDS cycle, but is brought down about 10\% during each discharge between cycles. The entire operating range for these cells (10\%-90\% SOC, delineated on the figure as the region between the thin dashed lines) is excited during the cell test.

\section{Comparing Maximum Power Calculations}

The PNGV HPPC power-estimation method gives a result that is a function of only SOC. Therefore, it is possible to graph available power versus SOC to summarize the algorithm calculations. The first method proposed in this paper is also possible to display in this way. Estimated power is only a function of SOC, $\partial \mathrm{OCV} / \partial z$ (also a function of SOC), and static limits on maximum current and power. The final method, however, depends on all states of the system dynamically. Two systems at the same state of charge, but with different voltage time-constant state values or hysteresis state levels will have different amounts of power available. To compare power computed by the three methods, dynamic tests must be conducted.

For the following results, we assume a pack of LiPB cells with $n_{s}=40$ and $n_{p}=1$. The data to fit the models was collected from prototype handmade cells jointly developed by LG Chem (Daejeon, Korea) and Compact Power Inc. (Monument, CO). Limits for the power calculations are listed in Table I. Each cell has a nominal capacity of $7.5 \mathrm{Ah}$ and $\Delta t$ was $10 \mathrm{~s}$ for both charge and discharge.

First, the PNGV HPPC method and Method I from this paper are compared in Fig. 6. The black curves correspond tou charge power and the gray curves correspond to discharge power. Note that the absolute value of power is plotted to avoid confusion due to sign conventions. First considering the calculations of charge power, we see that the PNGV HPPC method produces similar values to Method I in the mid-SOC range. The slight differences are due to the fact that the 10 -s $R^{\text {chg }}$ value used for the PNGV 


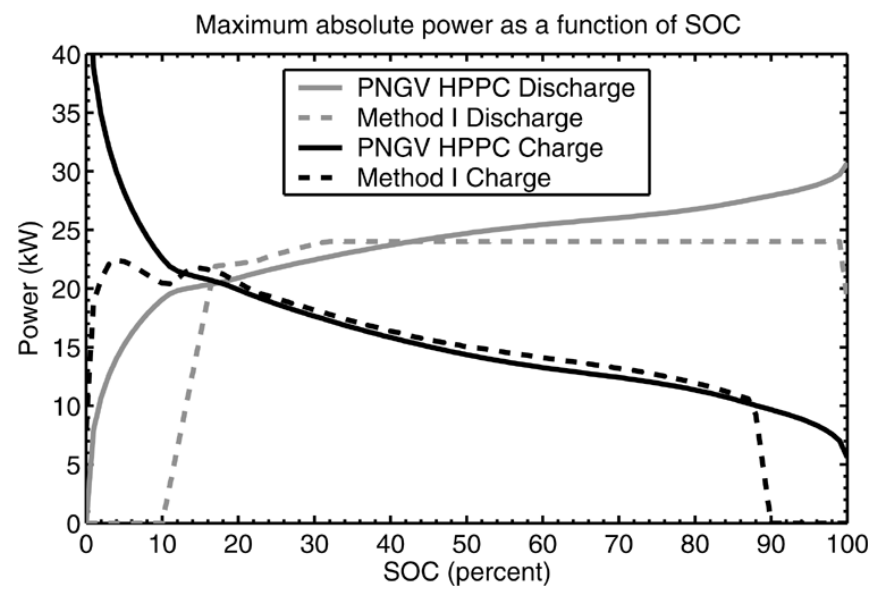

Fig. 6. Comparing static maximum power calculations as functions of SOC for the PNGV HPPC method and Method I of this paper.

method and the derivative-modified $R^{\text {chg }}$ for Method I are not identical. Outside the mid-SOC range, we see that Method I ramps power down in the neighborhood of $z_{\max }$ to avoid overcharging the cell, whereas the PNGV method has no such limit. At very low SOCs, the PNGV method overpredicts how much power is available since there are no current limits applied to the calculation. The Method I estimate is automatically lower due to the large derivative in the denominator of the calculation. This causes an anomaly near zero SOC, where the method under-predicts the available charge power. However, since the cell will not be operated in this range, this is not a concern.

Considering now the discharge power curves, we again see that Method I imposes limits on discharge power to ensure that the cell is not undercharged, whereas the PNGV method does not. In the SOC range from about $15 \%$ to $35 \%$, the two methods predict similar powers. For SOC above about 35\%, the power predicted by Method I saturates, because the maximum discharge current limit of 200 A has been reached. The PNGV method does not consider this limit. At SOC around 99\%, we again see an anomaly in the Method I calculation where power is underestimated due to the large derivative term. As before, we are not concerned by this glitch, since the cell will not be operated in this range.

The three methods described in this paper were used to estimate power for the cell undergoing the dynamic UDDS cell test whose voltage trace is shown in Fig. 3 and whose SOC trace is shown in Fig. 5. The discharge power estimates are plotted in Fig. 7 and the charge power estimates are plotted in Fig. 9. Again, the absolute value of power is plotted.

In the discussion that follows, we consider the results of Method II to be the "true" capability of the cell. We justify this assumption by the fidelity of the cell model's voltage estimates, as supported by the data in Fig. 4. In Fig. 7, we see that the three methods produce similar estimates. In particular, Methods I and II appear to be nearly identical when viewed at this scale. At high SOCs, the PNGV HPPC method predicts higher power than is actually available (by as much as 9.8\%), and at low SOCs the PNGV HPPC method underpredicts the available power. Only the methods introduced in this paper include SOC bounds, which explain why their predictions are so different

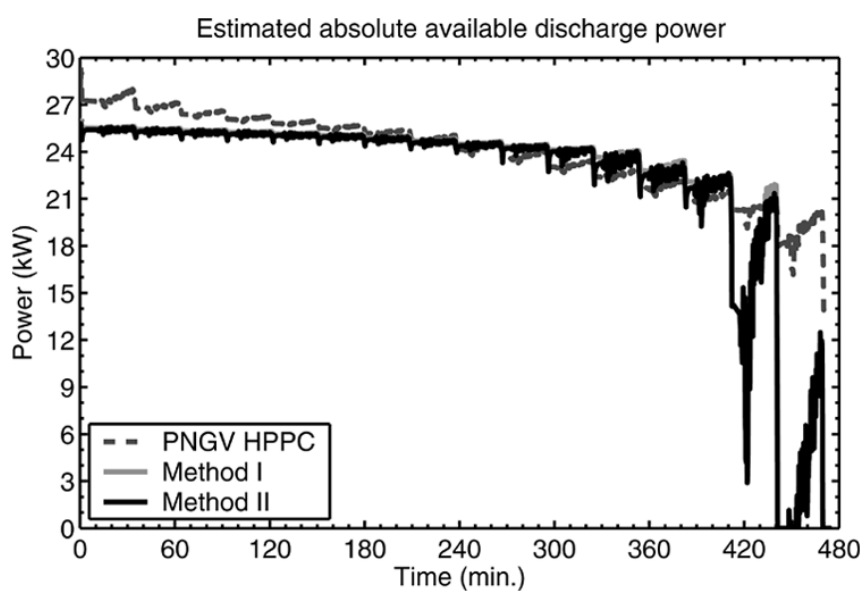

Fig. 7. Discharge power capability estimates for cell cycle test comprising 16 UDDS cycles over an SOC range of $90 \%$, down to $10 \%$.

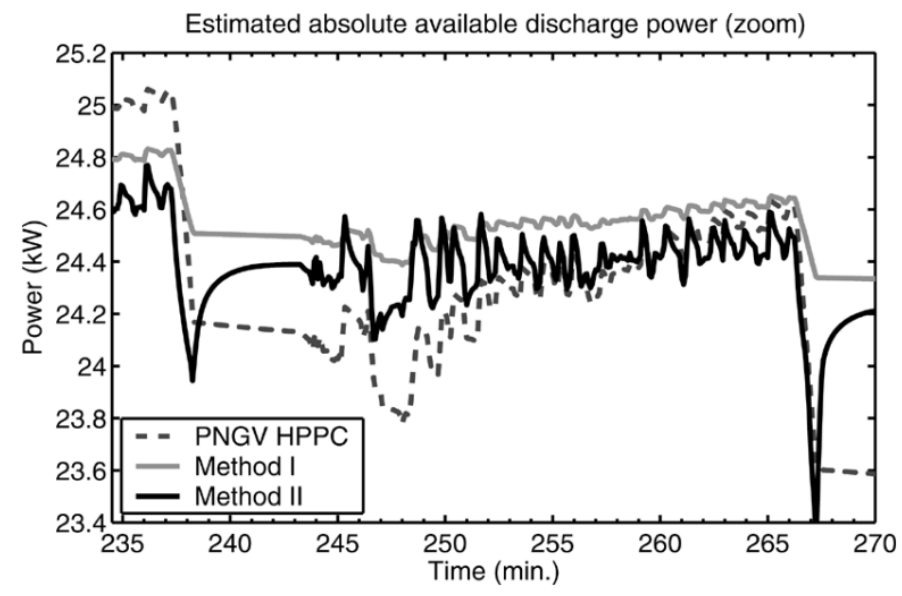

Fig. 8. Discharge power capability estimates for cell cycle test comprising 16 UDDS cycles over an SOC range of $90 \%$ down to $10 \%$.

from the PNGV HPPC estimates at low SOC. If the vehicle controller were to discharge at the rates predicted by the PNGV HPPC method, the cell would be overdischarged in some cases (lowering its lifetime) and underutilized in other cases. Fig. 8 zooms in on Fig. 7 (the same region shown as in Fig. 4) to show greater detail. In this region, the three methods produce nearly identical predictions. A notable feature of Method II, however, is that it takes into account the entire dynamics of the cell when making a prediction. Therefore, the strong discharges at around time 237 and 267 min draw the cell voltage down and allows less discharge power than the other two methods, which only consider SOC when making their estimate.

The three methods are also compared with respect to charge power, shown in Fig. 9. At this scale, the estimates appear nearly identical. Again, the PNGV HPPC method does not consider SOC limits, so it overpredicts charge power at high SOCs. It also overpredicts power at low SOCs, as it ignores the increase to charge resistance at low SOC. A zoom of this plot is shown in Fig. 10, which accentuates the differences between the predictions. Here, we see that the strong discharges at around time 237 and 267 min allow for greater charging power, as the voltage will not quickly change. 


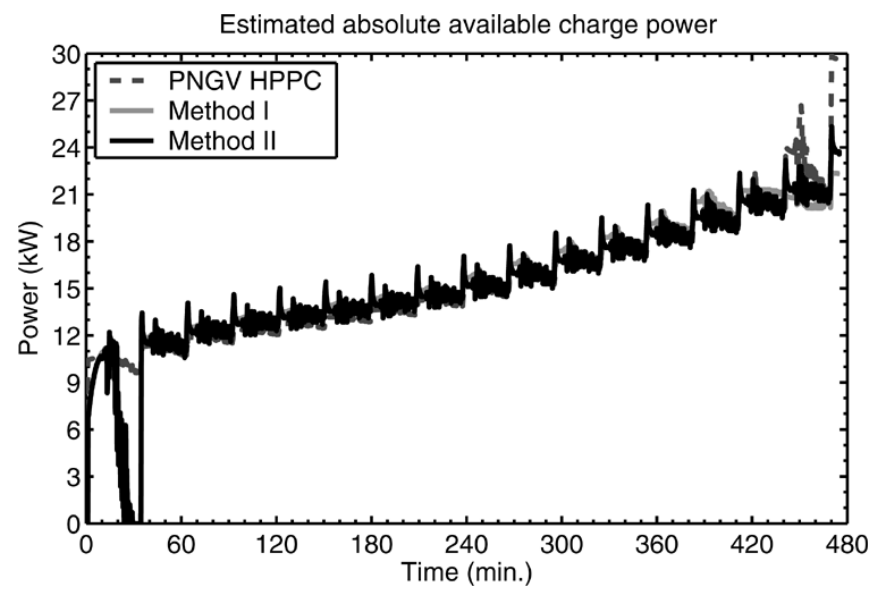

Fig. 9. Charging power capability estimates for cell cycle test comprising 16 UDDS cycles over an SOC range of $90 \%$ down to $10 \%$.

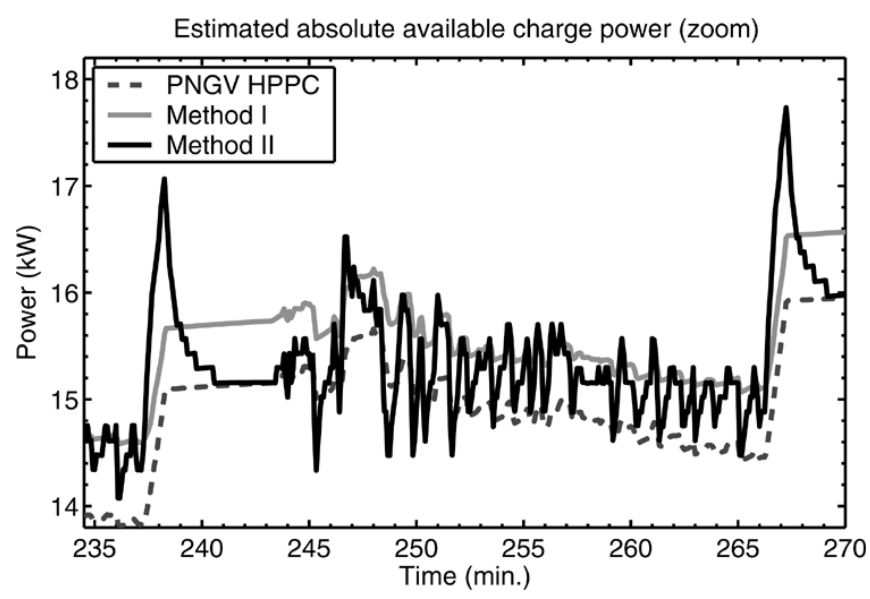

Fig. 10. Charging power capability estimates for cell cycle test comprising 16 UDDS cycles over an SOC range of $90 \%$ down to $10 \%$.

\section{CONCLUSION}

In this paper, we have presented two new methods to predict battery discharge and charge power that incorporate voltage, state of charge, power and current design constraints; work for a user-specified prediction horizon $\Delta t$; and are more robust and precise than the PNGV HPPC method. The results indicate that the two methods produce very similar results to each other. In most circumstances, Method I is acceptable and requires minimal computation. The PNGV HPPC method might be adapted to give results closely approximated by Method I if its resistance values are tabulated as functions of SOC, but then it would have no computational or storage advantage.
If very precise estimates are desired, then Method II should be used. It requires significantly more computation and a good cell model. If a Kalman filter is being used to estimate SOC, then the cell model will already be present and the state will be available for use. Method II produces dynamic power estimates, is able to take advantage of recent strong discharges to increase the temporary available charge power, and is able to take advantage of recent strong charges to increase temporary available discharge power.

\section{ACKNOWLEDGMENT}

The use of company facilities and many enlightening discussions with Dr. M. Alamgir, Dr. D. Rivers, and others are gratefully acknowledged. The author would also like to thank the reviewers for their corrections and helpful suggestions.

\section{REFERENCES}

[1] "PNGV Battery Test Manual," U.S. Dept. Energy, Idaho Nat. Eng. Environ. Lab., Rev. 3, DOE/ID-10 597, 2001.

[2] G. L. Plett, "Extended Kalman filtering for battery management systems of LiPB-based HEV battery packs-Part 1: Background," J. Power Sources, vol. 134, no. 2, pp. 252-261, 2004.

[3] — - "Extended Kalman filtering for battery management systems of LiPB-based HEV battery packs-Part 2: Modeling and identification," J. Power Sources, vol. 134, no. 2, pp. 262-276, 2004.

[4] - "Extended Kalman filtering for battery management systems of LiPB-based HEV battery packs-Part 3: State and parameter estimation," J. Power Sources, vol. 134, no. 2, pp. 277-292, 2004.

[5] - LiPB dynamic cell models for Kalman-filter SOC estimation. presented at Proc. 19th Electric Vehicle Symp. (EVS19)

[6] - Kalman-filter SOC estimation for LiPB HEV cells. presented at Proc. 19th Electric Vehicle Symp. (EVS19)

[7] - Advances in EKF SOC estimation for LiPB HEV battery packs. presented at Proc. 20th Electric Vehicle Symp. (EVS20)

[8] J. E. Marsden and A. J. Tromba, Vector Calculus, 3rd ed. San Francisco, CA: Freeman, 1988, pp. 243-247.

[9] C.-T. Chen, Linear System Theory and Design, 3rd ed. London, U.K.: Oxford Univ. Press, 1999.

[10] W. H. Press, S. A. Teukolsky, W. T. Vetterling, and B. P. Flannery, Numerical Recipes in C, 2nd ed. Cambridge, U.K.: Cambridge Univ. Press, 1992.

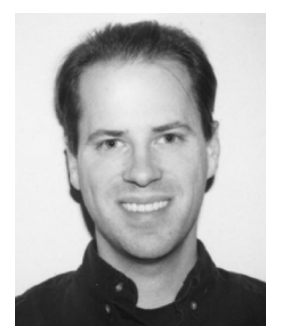

Gregory L. Plett (S'97-M'98-SM'02) was born in Ottawa, ON, Canada, in 1968. He received the B.Eng. degree in computer systems engineering (with high distinction) from Carleton University, Ottawa, in 1990 and the M.S. and Ph.D. degrees in electrical engineering from Stanford University, Stanford, CA, in 1992 and 1998, respectively.

He currently is an Assistant Professor of Electrical and Computer Engineering with the University of Colorado, Colorado Springs. He also is a Consultant to Compact Power Inc., Monument, $\mathrm{CO}$. 